\title{
Current concepts in management of $\mathrm{ACJ}$ injuries
}

\author{
Akshay Phadke*, Nik Bakti, Rajesh Bawale, Bijayendra Singh \\ Dept of Trauma \& Orthopaedics, Medway NHS Foundation Trust, United Kingdom
}

\section{A R T I C L E I N F O}

\section{Article history:}

Received 24 February 2019

Received in revised form

24 March 2019

Accepted 26 March 2019

Available online 1 April 2019

\section{Keywords:}

Acromioclavicular dislocation

Rockwood classification

Coracoclavicular ligament reconstruction

Hookplate

Arthroscopically assisted acromioclavicular

reconstruction

\begin{abstract}
A B S T R A C T
Acromioclavicular joint injury is common in young individuals who suffer direct trauma to the shoulder. Treatment of acromioclavicular dislocation is controversial with regards to the indication of operative management, timing of surgery, whether to perform open or arthroscopic surgery, method of stabilisation (rigid or non-rigid) and type of graft used for repair or reconstruction. Current evidence supports conservative management for Rockwood types I and II, while types IV, V and VI benefit from surgery. The optimal management of type III injuries in high demand patients remains contentious. Surgical options include acromioclavicular fixation, coracoclavicular fixation and coracoclavicular ligament reconstruction. Few studies with a low level of evidence suggest arthroscopic techniques and anatomical ligament reconstruction have better outcomes when compared to older techniques of rigid coracoclavicular fixation. The aim of this article is to look at the current evidence and address these controversial issues.
\end{abstract}

๑) 2019 Delhi Orthopedic Association. All rights reserved.

\section{Introduction}

The acromio-clavicular (AC) joint is a di-arthrodial joint which acts as a link between axial and appendicular skeleton and contributes to the complex pattern of movement of the shoulder joint. $\mathrm{AC}$ joint injuries are most commonly seen in young adults and about $43 \%$ of injuries occur in second decade of life. AC joint injuries are 5 times more common in men and often involve contact sports. ${ }^{1,2}$ Despite being a common injury, there is no consensus regarding optimal management of $\mathrm{AC}$ joint dislocation. In recent years numerous techniques have been developed and described. Controversy exists regarding the choice of surgical procedure for treatment of injuries and the timing of surgery. ${ }^{3,4}$ The following work attempts to review literature regarding recent developments in the treatment of acute AC joint injuries and provide recommendations based on sound clinical evidence.

\subsection{Anatomy of AC joint and classification of its injuries}

The AC joint is a synovial joint and its articular surface is made up of hyaline cartilage with an interposed disc of cartilage identical to a meniscus. This meniscus undergoes attrition over-time and is practically absent by the fourth decade of life. The articular surface

\footnotetext{
* Corresponding author.

E-mail address: akshay.phadke@nhs.net (A. Phadke).
}

area is small, whereas load transmitted is high. Cadaveric study of $A C$ joint morphology has demonstrated three main types of $A C$ joints 1)Flat, 2)Oblique and 3)Curved (Fig. 1). ${ }^{5}$ The forces are uniformly distributed in a flat acromion, however a curved or an oblique joint may have asymmetric stress transfer promoting damage to the articular cartilage.

The AC joint is stabilised by the AC ligaments which are thickenings in the joint capsule and the coraco-clavicular (CC) ligaments. The joint experiences significant translational forces in the anteroposterior and superolateral directions due to weight of the arm and muscle pull. The AC ligament provides about $90 \%$ of the AP stability and $20-50 \%$ of resistance to superior migration. The CC ligament consists of the conoid ligament medially and the trapezoid laterally. They are the primary restraint to inferior and medial translation of the acromion in respect to the clavicle. ${ }^{6}$

\section{Classification}

The sequential pattern of injury beginning with the AC ligaments, progressing to the coraco-clavicular ligaments and finally involving the deltoid and trapezial muscles was originally described by Cadenat. ${ }^{7}$ Rockwood described a classification which is most commonly accepted presently (Fig. 2 ). ${ }^{2}$ In type I injury, the AC and CC ligaments are all intact and the radiographic examination is normal. In type II injuries, the force of trauma is severe enough to rupture the AC ligaments, yet not severe enough to rupture the CC ligaments. Radiographs may show slight elevation of 


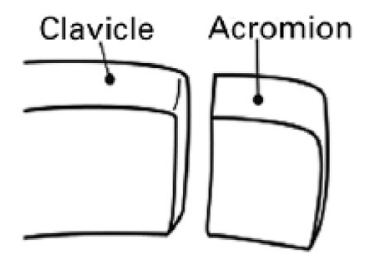

Flat

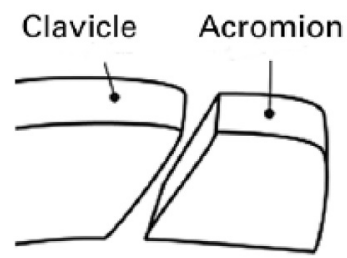

\section{Oblique}
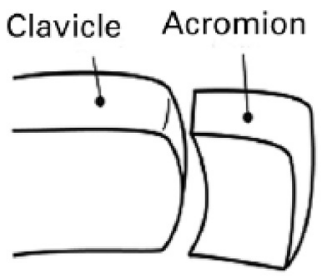

\section{Curved}

Fig. 1. Coronal CT images showing three distinct morphological shapes ${ }^{5}$.

the lateral end of clavicle. Type III injuries involve complete disruption of both AC and CC ligaments without severe disruption of deltoid or trapezial fascia. The clavicle is unstable in both horizontal and vertical plane, and stress view radiographs are abnormal with a $100 \%$ separation of the AC joint. In type IV injuries, the distal clavicle is displaced posteriorly into the trapezius and may tent the posterior skin. Type V is a more severe form of type III injury with disruption of trapezial and deltoid fascia, manifested by a 100-300\% increase in the coracoclavicular distance. Type VI is a complete dislocation with inferior displacement of the distal clavicle into a subacromial or subcoracoid position.

\subsection{Mechanism of injury}

The mechanism of injury is most often a direct fall on the outer aspect of shoulder with the arm in adduction which displaces the acromion inferiorly and medially. The acromioclavicular ligaments are the first to fail, allowing the load to be transmitted to the coracoclavicular ligaments. Disruption of coracoclavicular ligament leaves the trapezoid and deltoid muscles to act as restraints. Higher forces can lead to rupture of these muscle attachments as well. Associated injuries might include clavicular fractures and sternoclavicular joint injuries and should be screened for.

\subsection{Nonoperative}

Nonoperative management is indicated for types I and II AC dislocations. Early range-of motion exercises are encouraged, and daily activities resumed when pain permits. Treatment of type III dislocation is controversial. Several studies published in the 1980s support the use of conservative treatment over surgery with a good long-term outcome. ${ }^{8-10}$ However, the procedures included in the above studies, $\mathrm{K}$ wire fixation of $\mathrm{AC}$ joint and Bosworth method of CC fixation, are outdated and therefore seldom used today. Rawes and Dias published a long-term follow-up (average 12.5 years) of conservatively treated type III AC joint dislocations and found satisfactory outcomes in all except one patient. All their patients showed subluxated or dislocated joint and although anatomical reduction was not obtained results were favourable. ${ }^{10}$

Non-surgical management is usually not preferred for high grade injuries. However, Joukainen et al. published results at 18-20-year follow-up which showed no differences in type III and type $\mathrm{V}$ dislocations at the end of follow-up period. Again operative treatment consisted of transarticular pinning which is rarely performed these days. ${ }^{11}$

In a retrospective study, Dunphy et al. analysed patients with Type V Acromioclavicular injuries, most patients were able to return to work following conservative management at 6 months but

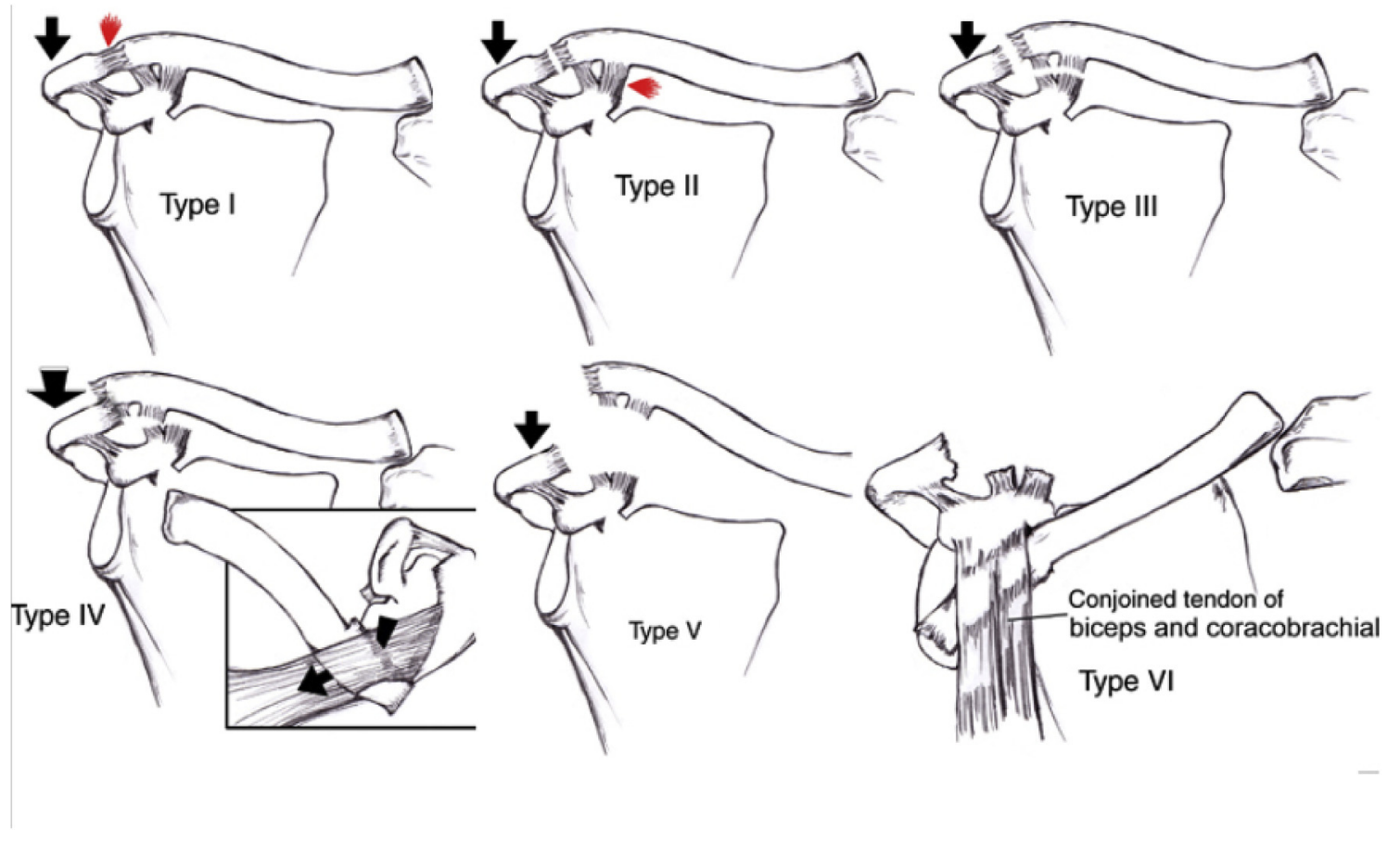

Fig. 2. Rockwood classification of Acromioclavicular joint dislocations. 
had limited functional outcome scores. When compared to surgical intervention, conservatively managed manual workers had early recovery and were back to work at average 4 weeks as against 11 weeks after surgery. ${ }^{12}$ However, Gstettner et al. reported favourable outcomes following surgery for Grade II A-C dislocations when compared to conservative management. ${ }^{13}$ There is lack of conclusive evidence for outcome of Rockwood types III - V, but overall early surgery might give a better outcome to select cohort of young and physically active individuals. ${ }^{14}$

\section{Acromioclavicular fixation}

Acromio-clavicular fixation allows healing of the disrupted coraco-acromial ligaments by restoring the distance between the clavicle and the coracoid. Satisfactory outcomes have been reported in literature with Kirchner Wire (K wire) fixation and ligament repair. However, the procedure is rarely used today because of high incidence of migration of $\mathrm{K}$ wires and loss of reduction. ${ }^{15,16}$

\subsection{Hook-plate}

Clavicle hook plates are anatomically pre-contoured plates with varying sizes and depths to accommodate individual anatomy. Clavicle is reduced by pushing it down manually and temporary fixation is done with $\mathrm{K}$ wires. Soft tissue is dissected posterior to the AC joint to make way for the hook. Using the plate with $15 \mathrm{~mm}$ hook depth, alignment is checked and if the realignment appears to be overcorrected, a plate with $18 \mathrm{~mm}$ hook depth is used. Before final fixation, shoulder is moved in abduction and external rotation to rule out impingement of the humeral head on the hook. Most manufacturers recommend removal of the hook plate after healing to prevent potential impingement and osteolysis of the acromion.

The hook plate provides a rigid construct and allows early mobilisation. Kinast et al. published a mid term follow-up of 313 patients with grade III-V lesions of AC joint and were able to demonstrate good results with a low (10.6\%) complication rate. ${ }^{17}$

Salem and Schmelz reported satisfactory outcomes in 2/23 patients with complete AC disruptions treated with hook plates. ${ }^{18}$

The disadvantage of using a hook plate is that the hook physically impinges on the subacromial structures and causes a subacromial erosion due to point loading on the under-surface of acromion.

Lin et al. were able to demonstrate impingement and rotator cuff lesions in $37.5 \%$ of their patients. Poor functional scores correlated with hardware associated impingement. The study also showed a $50 \%$ rate of acromial erosion. ${ }^{19}$

Zang et al. advocate early removal of the hook plate to prevent hook related complications. ${ }^{20}$ In their study group, the mean constant shoulder score was greater when plate was removed before 3 months after healing. The complication rates significantly increased with retained hardware and delayed removal of the plate when compared to early removal ( $50 \%$ and $22.5 \%$ vs $10 \%)^{21}$

In a comparative analysis of hook plate fixation with nonoperative management, constant score was significantly better in non-operative groups after 3 months but long term functional scores were not significantly different. ${ }^{22}$ Reduction maintained was better with use of plate, but this did not correlate with functional improvement. Based on the trial the authors could not recommend the use of hook plates as it did not improve the outcome.

\section{Coraco-clavicular fixation}

The coraco-clavicular distance can be maintained either by rigid fixation with a screw or the use of non-rigid synthetic materials. Bosworth described coraco-clavicular stabilisation with a lag screw. ${ }^{23}$ Rockwood and Young modified the technique to include CC ligament repair or reconstruction depending on the time of surgery. A bicortically placed screw provides about $80 \%$ more strength than the native ligament. ${ }^{24}$ However, this technique does not allow the clavicle to move in relation to the coracoid, increasing the chances of stress related loosening. Another disadvantage of the procedure is the necessity of a second procedure to remove the screw. ${ }^{23}$ A risk of loosening and loss of reduction up to $6 \%$ has been reported in recent literature. ${ }^{23}$

Non-rigid techniques have the advantage of allowing some clavicular motion and do not require a second surgery for removal of implants. Suture button fixation has been shown to provide biomechanical strength similar to the native CC ligament in vivo. A high failure rate has been reported with the use of single endobutton, with knot slippage, suture breakage and button migration. A prospective study of 9 patients with CC reconstruction with synthetic fibre resulted in high incidence of loss of reduction and ( 7 of 9) and high re-operative rate (4 of 9). The authors suggest high forces acting across the shoulder joint to be the probable cause of failure. ${ }^{25}$ Milewski et al. compared the results of anatomic CC reconstruction using a tendon graft and a cortical fixation button (CFB) with and without a coracoid tunnel. The coracoid tunnel group had $20 \%$ incidence of coracoid fractures and a $50 \%$ incidence of loss of reduction. The authors noted lower incidence of complications (coracoid fractures and loss of reduction) when the graft was looped around the base of coracoid. ${ }^{26}$ Martetschlager et al. reported an overall complication rate of $27.1 \%$ with a $83 \%$ construct survivorship at 2 years. $^{27}$

\section{Ligament reconstruction}

The Weaver-Dunn procedure involves stabilisation of the $A C$ joint by transfer of native CA ligament from acromion to the distal clavicle. The original technique also included excision of the distal clavicle. Initial reports from Weaver and Dunn revealed a 75\% goodexcellent outcome. ${ }^{28}$ Biomechanical studies have demonstrated that the native CA ligament is weakest amongst the various constructs tested. ${ }^{29}$ The initial strength of the CA ligament construct was found to be only a quarter of the native ligament and did not offer any anteroposterior stability. A recent systemic review Sood et al. found an unacceptable rate of recurrence of deformity when the CA ligament was used due to fixation related complications. ${ }^{30}$ When an open clavicle excision is performed, the surgeon should be aware of the high rates of complications, including infection, stiffness, continued AC joint sensitivity and scar hypertrophy ${ }^{31}$ and should be reserved only for chronic AC joint instability.

Recently autografts and allografts have gained popularity for anatomic reconstruction of AC joint. Jones et al. described this technique for the first time in 2001, and it involves creating two tunnels in clavicle at coronoid and trapezoid footprints and passing a tendon graft through each tunnel and under or through the base of coracoid. The remaining longer limb of the graft may be used to reconstruct the $A C$ ligaments. This anatomically recreates both the $\mathrm{AC}$ and CC ligaments of the joint. ${ }^{32}$

A cadaveric study by Mazocca et al. has demonstrated the superiority of anatomic ligament reconstruction when compared to the traditional Weaver Dunn technique and arthroscopic suture fixation. ${ }^{33}$

Tauber et al. compared anatomic semitendinous CC ligament reconstruction with modified Weaver Dunn procedure and found superior results of the anatomic technique. ${ }^{34}$

Synthetic material has been be used for anatomic CC and AC ligament reconstruction. Outcomes with LARS (Surgical implants and Devices, Arc Sur Tille, France) Ligament were found superior when compared to a dacron graft. ${ }^{35}$ 


\subsection{Surgilig ${ }^{\mathrm{TM}}$ reconstruction}

In 2001 the Nottingham shoulder unit developed a synthetic substitute for the coraco-clavicular ligament. ${ }^{36}$ The Surgilig ${ }^{\mathrm{TM}}$ is a braided polyester with two loops and can be used to treat both acute and chronic acromioclavicular dislocations. The procedure is simple and quick and involves passing the synthetic ligament around the coracoid and passing one loop through the other to secure it to the coracoid (Figs. 3 and 4). The other end is passed around the distal clavicle from posterior to the anterior direction and fixed to the clavicle with a screw. The synthetic ligament has good tensile strength, promotes tissue ingrowth and avoids sacrifice of the native coracoacromial ligament for reconstruction. ${ }^{36}$

Although there are several small studies which report outcomes with Surgilig TM reconstruction for chronic acromiocalviclular injuries, only two studies show the effective ness of this technique for acute injuries. Wright et al. reported high satisfaction rates (20/ $21)^{37}$ Mean abduction power of operated site was $82 \%$ of the normal. There was no infection, clavicular or coracoid fracture and none of the patient required implant removal.

Younis et al. found the Surgiligтм to be safe and effective in treatment of both chronic and acute acromioclaviclular joint dislocations grades $3-5 .^{38}$

\subsection{Arthroscopic techniques}

With the advent of arthroscopy, new techniques and implants have been developed allowing a minimally invasive, and arthroscopically assisted surgery. Arthroscopic CC stabilisation was first

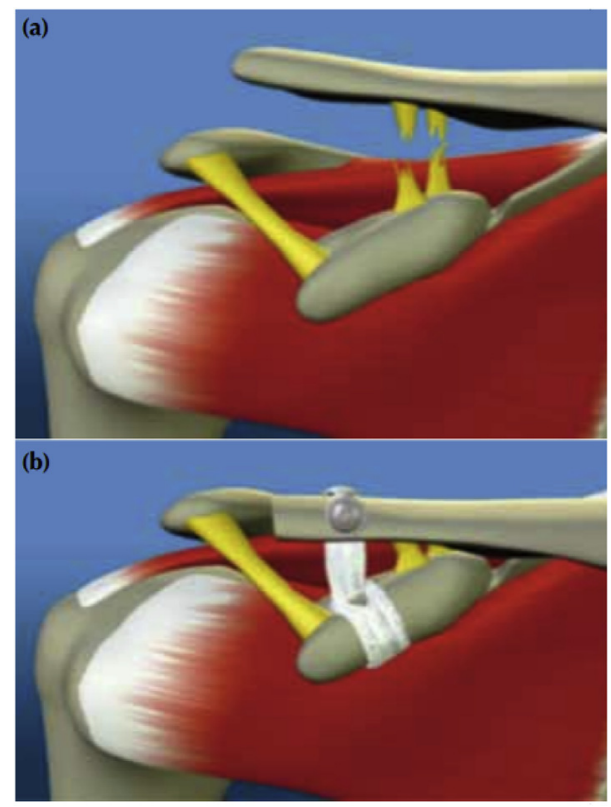

Fig. 3. a) Rupture of coracoclavicular ligaments, b) Reconstruction with prosthetic ligament.

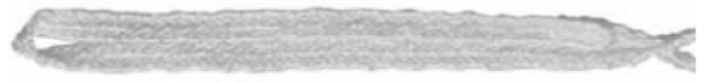

Fig. 4. Synthetic double loop ligament. described by Wolf and Pennington, ${ }^{39}$ who used wire cerclages and reported satisfactory results in $81 \%$ of their patients. Arthroscopic modification of Bosworth's technique was described by Rolla et al. with excellent early outcomes. ${ }^{21} \mathrm{~A}$ minimally invasive technique for stabilisation of CC interval with suture anchors was described by Elser et al. Twelve of their 13 patients had a satisfactory outcome.

Recently the use of TightRope ${ }^{\mathrm{TM}}$ (Arthrex, Naples) has gained popularity for ACJ repair. Two titanium buttons are connected by a No. 5 FibreWire (Arthrex) suture and are introduced through a $4 \mathrm{~mm}$ CC drill hole with a special guiding device. The inferior subcoracoid button is flipped and the TightRope is secured by tying a knot over the clavicle. ${ }^{40} \mathrm{~A}$ two year follow-up has been published with TightRope fixation of AC joint. ${ }^{20}$ Six of their 24 patients (25\%) had a partial loss of reduction and inferior Constant scores compared to the rest of the patients. The authors describe two reasons for failure of the construct. Early failure can be caused by cutting of the fibre loop through the cortex of the clavicle. Eccentric drilling through the anterior cortex might be one of the important causes. Partial loss of reduction was seen with clavicular osteolysis associated with the clavicular button.

A second generation of the TightRope system allows anatomical CC fixation with two TightRopes. Biomechanical studies have shown that anatomic double bundle augmentation offers better horizontal stability and strength comparable to the native CC ligament complex. $^{41}$

A complete diagnostic arthroscopy of the gleno-humeral joint is recommended before ACJ stabilisation is performed. High grade AC dislocations have been shown to be associated with traumatic concomitant glenohumeral pathologies in about $15 \%$ of cases. ${ }^{42} \mathrm{~A}$ combined or an all-arthroscopic technique allows accurate diagnosis and treatment of intra-articular pathologies.

The technique of double button fixation can be carried out using a mini-open technique as well using the MINAR system. ${ }^{43}$ With a mini-open technique it is possible to have a good visualisation of the coracoid base which helps in accurate positioning of the coracoid drill holes. Secondly, delto-trapezoidal fascia can be repaired which is not possible with all arthroscopic techniques.

\subsection{Timing of surgery}

There are some contradictions regarding the definition of a chronic AC joint injury and the timing of surgery. For comparing the outcomes of early and delayed surgery it is essential to establish a dividing line between the two stages. The dividing line has been variously defined as 3 weeks, ${ }^{44} 4$ weeks ${ }^{45}$ and 6 weeks ${ }^{46}$ by different authors. Pain from an acute injury subsides by three weeks after acromio-clavicular dislocation ${ }^{2}$ and so 3 weeks seems to be a relevant dividing line. Song et al. compared the results of early and delayed surgical treatment of complete acomio-clavicular injuries. ${ }^{47}$ The study found that early surgical treatment results in a superior outcome and better reduction as compared to delayed surgery.

\section{Conclusion}

In the recent years there has been an exponential increase in the number of publications on repair and reconstruction of AC joint. ${ }^{48}$ However, most of the recent studies are case series and lack longterm follow-up. Lack of standardisation in terms of population homogeneity, severity and chronicity of the injury prevents drawing up recommendations based on sound evidence. Significant questions still remain unanswered, with regards to type of graft to be used, number of ligaments reconstructed, number of drill holes made and their position and finally the technique of reconstruction.

Evidence suggests that compared to conservative treatment, 
surgical management of type III AC joint injuries have a better cosmetic and radiological outcome and comparable functional outcome. ${ }^{8-10}$ However, the rate of complications and time off work is significantly higher with operative management. Early operative intervention results in better functional outcome and reduces the risk of infection and loss of reduction.

There is consensus in the literature that type IV injuries should be managed surgically. Conservative management is associated with high level of pain due to AC instability. ${ }^{40,49} \mathrm{~A}$ high rate of persistent pain was reported after isolated CC stabilisation using TightRope due to horizontal ACJ instability in $43 \%$ cases. $^{50}$ Surgical treatment should focus on stabilisation of both vertical and horizontal components to improve the clinical outcome.

The selection of graft depends on the chronicity of the injury. Evidence suggests that biological augmentation is required in chronic cases to enhance the healing of the torn ligaments. ${ }^{51} \mathrm{Su}-$ periority of anatomic reconstruction with double tendon graft has been validated by both biomechanical and clinical studies. ${ }^{41}$

Arthroscopically assisted AC reconstruction offers the advantages of assessment of glenohumeral joint, better visualisation of the coracoid process and is minimally invasive. However, it has a steep learning curve and currently no evidence to support it over open surgery.

\section{Declaration of interest}

None.

\section{References}

1. Pallis M, et al. Epidemiology of acromioclavicular joint injury in young athletes. Am J Sports Med. 2012;40(9):2072-2077. https://doi.org/10.1177/ 0363546512450162.

2. Rockwood CA. The Shoulder. Saunders/Elsevier; 2009.

3. Cisneros LN, Reiriz JS. Management of chronic unstable acromioclavicular joint injuries. J Orthop Traumatol. 2017;18(4):305-318. https://doi.org/10.1007/ s10195-017-0452-0.

4. Modi CS, et al. Controversies relating to the management of acromioclavicular joint dislocations. The Bone \& Joint Journal. 2013;95-B(12):1595-1602. https:// doi.org/10.1302/0301-620X.95B12.31802.

5. Colegate-Stone T, et al. Classification of the morphology of the acromioclavicular joint using cadaveric and radiological analysis. Consult Orthop Surg J Bone Joint Surg [Br]. 2010;92(5):92-743. https://doi.org/10.1302/0301620X.92B5.

6. Mazzocca AD, et al. Biomechanical and radiographic analysis of partial coracoclavicular ligament injuries. Am J Sports Med. 2008;36(7):1397-1402. https://doi.org/10.1177/0363546508315200.

7. C F. The treatment of dislocations and fractures of the outer end of the clavicle. Int Clin. 1917;1:145-169. Available at: https://ci.nii.ac.jp/naid/10007739385/. Accessed October 28, 2018.

8. Galpin RD, Hawkins RJ, Grainger RW. A comparative analysis of operative versus nonoperative treatment of grade III acromioclavicular separations. Clin Orthop Relat Res. 1985;193:150-155. Available at: http://www.ncbi.nlm.nih. gov/pubmed/3971615. Accessed October 11, 2018.

9. Larsen E, Bjerg-Nielsen A, Christensen P. 'Conservative or surgical treatment of acromioclavicular dislocation. A prospective, controlled, randomized study. American volume J Bone Jt. Surg. 1986;68(4):552-555. Available at: http:// www.ncbi.nlm.nih.gov/pubmed/3514625. Accessed October 11, 2018.

10. Rawes ML, Dias JJ. Long-term results of conservative treatment for acromioclavicular dislocation. British volume J Bone Jt. Surg. 1996;78(3):410-412. Available at: http://www.ncbi.nlm.nih.gov/pubmed/8636176. Accessed October 11, 2018.

11. Joukainen A, et al. Results of operative and nonoperative treatment of rockwood types III and V acromioclavicular joint dislocation: a prospective, randomized trial with an 18- to 20-year follow-up. SAGE Publications Orthop J Sports Med. 2014;2(12). https://doi.org/10.1177/2325967114560130, 2325967114560130.

12. Dunphy TR, et al. Functional outcomes of type $\mathrm{V}$ acromioclavicular injuries with nonsurgical treatment. J Am Acad Orthop Surg. 2016;24(10):728-734. https://doi.org/10.5435/JAAOS-D-16-00176.

13. Gstettner C, et al. Rockwood type III acromioclavicular dislocation: surgical versus conservative treatment. J Shoulder Elb Surg. 2008;17(2):220-225. https://doi.org/10.1016/j.jse.2007.07.017.

14. Porschke F, et al. Sports activity after anatomic acromioclavicular joint stabilisation with flip-button technique. Knee Surg Sports Traumatol Arthrosc: Off J ESSKA. 2017;25(7):1995-2003. https://doi.org/10.1007/s00167-016-4287-7.
15. Leidel BA, et al. Consistency of long-term outcome of acute rockwood grade III acromioclavicular joint separations after K-wire transfixation. J Trauma Inj Infect Crit Care. 2009;66(6):1666-1671. https://doi.org/10.1097/TA.0b013e31818c1455.

16. Lizaur A, Sanz-Reig J, Gonzalez-Parreño S. 'Long-term results of the surgical treatment of type III acromioclavicular dislocations. British volume J Bone Jt. Surg. 2011;93-B(8):1088-1092. https://doi.org/10.1302/0301-620X.93B8.26775.

17. Kienast $B$, et al. Mid-term results after operative treatment of rockwood grade III-V acromioclavicular joint dislocations with an AC-hook-plate. Eur J Med Res BioMed Central. 2011;16(2):52-56. https://doi.org/10.1186/2047-783X-16-252.

18. Salem KH, Schmelz A. Treatment of tossy III acromioclavicular joint injuries using hook plates and ligament suture. J Orthop Trauma. 2009;23(8):565-569. https://doi.org/10.1097/BOT.0b013e3181971b38.

19. Lin H-Y, et al. Clavicular hook plate may induce subacromial shoulder impingement and rotator cuff lesion - dynamic sonographic evaluation. J. Orthop Surgery Res BioMed Central. 2014;9(1):6. https://doi.org/10.1186/ 1749-799X-9-6.

20. Zhang L-F, et al. Arthroscopic fixation of acute acromioclavicular joint disruption with TightRope ${ }^{\mathrm{TM}}$ : outcome and complications after minimum 2 (2-5) years follow-up. J Orthop Surg. 2017;25(2). https://doi.org/10.1177/ 2309499016684493, 230949901668449.

21. Rolla PR, Surace MF, Murena L. Arthroscopic treatment of acute acromioclavicular joint dislocation. Arthrosc J Arthrosc R Surg Offic Publ Arthrosc Assoc North Am Int Arthrosc Assoc. 2004;20(6):662-668. https://doi.org/10.1016/ j.arthro.2004.03.012.

22. Canadian Orthopaedic Trauma Society. Multicenter randomized clinical trial of nonoperative versus operative treatment of acute acromio-clavicular joint dislocation. J Orthop Trauma. 2015;29(11):479-487. https://doi.org/10.1097/ BOT.0000000000000437.

23. Esenyel CZ, et al. Coracoclavicular ligament repair and screw fixation in acromioclavicular dislocations. Acta Orthop Traumatol Turcica. 2010;44(3): 194-198. https://doi.org/10.3944/AOTT.2010.2329.

24. Harris RI, et al. Structural properties of the intact and the reconstructed coracoclavicular ligament complex. Am J Sports Med. 2000a;28(1):103-108. https://doi.org/10.1177/03635465000280010201.

25. Singh B, Mohanlal P, Bawale R. Early failure of coracoclavicular ligament reconstruction using TightRope system; 2016. undefined. Available at: https:// www.semanticscholar.org/paper/Early-failure-of-coracoclavicular-ligamentusing-Singh-Mohanlal/3bc2f34494ae55f8aeb1a4f3e62379ceffdc1e04. Accessed February 22, 2019.

26. Milewski MD, et al. Complications related to anatomic reconstruction of the coracoclavicular ligaments. Am J Sports Med. 2012;40(7):1628-1634. https:// doi.org/10.1177/0363546512445273.

27. Martetschläger $\mathrm{F}$, et al. Complications after anatomic fixation and reconstruction of the coracoclavicular ligaments. Am J Sports Med. 2013;41(12): 2896-2903. https://doi.org/10.1177/0363546513502459.

28. Weaver JK, Dunn HK. Treatment of acromioclavicular injuries, especially complete acromioclavicular separation. J Bone Jt. Surg. 1972;54(6):1187-1194. Available at: http://www.ncbi.nlm.nih.gov/pubmed/4652050. Accessed March 23, 2019.

29. Harris RI, et al. Structural properties of the intact and the reconstructed coracoclavicular ligament complex. Am J Sports Med. 2000b;28(1):103-108. https://doi.org/10.1177/03635465000280010201.

30. Sood A, Wallwork N, Bain GI. Clinical results of coracoacromial ligament transfer in acromioclavicular dislocations: a review of published literature. Int J Shoulder Surg. 2008;2(1):13-21. https://doi.org/10.4103/0973-6042.39582. Wolters Kluwer - Medknow Publications.

31. Chronopoulos E, et al. 'Complications after open distal clavicle excision. Clin Orthop R Res Assoc Bone Jt Surg. 2008;466(3):646-651. https://doi.org/10.1007/ s11999-007-0084-4.

32. Jones HP, Lemos MJ, Schepsis AA. Salvage of failed acromioclavicular joint reconstruction using autogenous semitendinosus tendon from the knee. Am J Sports Med. 2001;29(2):234-237. https://doi.org/10.1177/03635465010290022001. SAGE PublicationsSage CA: Los Angeles, CA.

33. Mazzocca AD, et al. A biomechanical evaluation of an anatomical coracoclavicular ligament reconstruction. Am J Sports Med. 2006;34(2):236-246. https://doi.org/10.1177/0363546505281795.

34. Tauber M, et al. Semitendinosus tendon graft versus a modified weaver-dunn procedure for acromioclavicular joint reconstruction in chronic cases. Am J Sports Med. 2009;37(1):181-190. https://doi.org/10.1177/0363546508323255.

35. Fauci F, et al. Surgical treatment of chronic acromioclavicular dislocation with biologic graft vs synthetic ligament: a prospective randomized comparative study. J Orthop Traumatol: Offic J Ital Soc Orthop Traumatol. 2013;14(4): 283-290. https://doi.org/10.1007/s10195-013-0242-2. Springer.

36. Jeon I-H, et al. Chronic acromioclavicular separation: the medium term results of coracoclavicular ligament reconstruction using braided polyester prosthetic ligament. Injury. 2007;38(11):1247-1253. https://doi.org/10.1016/ j.injury.2007.05.019.

37. Wright J, et al. Stabilisation for the disrupted acromioclavicular joint using a braided polyester prosthetic ligament. Hong Kong J Orthop Surg. 2015 Aug;23(2):223-228. Available at: https://pdfs.semanticscholar.org/9cfb/ 1e2bd70f13d367891267b691296ff9092fb3.pdf . Accessed January 6, 2019.

38. Younis F, et al. Operative versus non-operative treatment of grade III acromioclavicular joint dislocations and the use of SurgiLig: a retrospective review. Ortopedia Traumatologia Rehabilitacja. Index Copernicus. 2017;19(6):523-530. 
https://doi.org/10.5604/01.3001.0010.8043.

39. Wolf EM, Pennington WT. Arthroscopic reconstruction for acromioclavicula joint dislocation. Arthrosc J Arthrosc Relat Surg. 2001;17(5):558-563. https:// doi.org/10.1053/jars.2001.23578. Elsevier.

40. Tauber M. Management of acute acromioclavicular joint dislocations: current concepts. Arch Orthop Trauma Surg. 2013;133(7):985-995. https://oi.org 10.1007/s00402-013-1748-z.

41. Zooker CC, et al. TightRope versus fiber mesh tape augmentation of acromioclavicular joint reconstruction. Am J Sports Med. 2010;38(6):1204-1208. https://doi.org/10.1177/0363546509359064.

42. Pauly S, et al. Prevalence of concomitant intraarticular lesions in patients treated operatively for high-grade acromioclavicular joint separations. Knee Surg Sports Traumatol Arthrosc. 2009;17(5):513-517. https://doi.org/10.1007| s00167-008-0666-Z

43. Petersen W, et al. Minimalinvasive Akromioklavikulargelenkrekonstruktion (MINAR). Operat Orthop Traumatol. 2010;22(1):52-61. https://doi.org/10.1007| s00064-010-3004-4.

44. Motta P, et al. Acromioclavicular motion after surgical reconstruction. Knee Surg Sports Traumatol Arthrosc. 2012;20(6):1012-1018. https://oi.org 10.1007/s00167-011-1627-5.

45. Ejam S, Lind T, Falkenberg B. Surgical treatment of acute and chronic acromioclavicular dislocation Tossy type III and V using the Hook plate. Acta Orthop Belg. 2008;74(4):441-445. Available at: http://www.ncbi.nlm.nih.gov pubmed/18811024. Accessed March 23, 2019.

46. Rolf $\mathrm{O}$, et al Acromioclavicular dislocation Rockwood III-V: results of early versus delayed surgical treatment. Arch Orthop Trauma Surg. 2008;128(10): 1153-1157. https://doi.org/10.1007/s00402-007-0524-3.

47. Song T, Yan X, Ye T. Comparison of the outcome of early and delayed surgical treatment of complete acromioclavicular joint dislocation. Knee Surg Sports Traumatol Arthrosc. 2016;24(6):1943-1950. https://doi.org/10.1007/s00167014-3225-9.

48. Beitzel K, et al. 'Current concepts in the treatment of acromioclavicular joint dislocations. Arthrosc J Arthrosc Relat Surg. 2013;29(2):387-397. https:// doi.org/10.1016/j.arthro.2012.11.023.

49. Echo BS, Donati RB, Powell CE. 'Bipolar clavicular dislocation treated surgically. A case report. American volume J Bone Jt. Surg. 1988;70(8):1251-1253. Available at: http://www.ncbi.nlm.nih.gov/pubmed/3417712 . Accessed November 4, 2018.

50. Scheibel M, et al. Arthroscopically assisted stabilization of acute high-grade Acromioclavicular joint separations. Am J Sports Med. 2011;39(7):1507-1516. https://doi.org/10.1177/0363546511399379.

51. Rasmont $\mathrm{Q}$, et al. Is conservative treatment still defensible in grade III acromioclavicular dislocation? Are there predictive factors of poor outcome? Acta Orthop Belg. 2015;81(1):107-114. Available at: http://www.ncbi.nlm.nih.gov/ pubmed/26280863. Accessed November 4, 2018. 